\title{
Activación conductual para el abandono del tabaco en una persona con depresión: un estudio de caso.
}

\section{Behavioural activation for smoking cessation and depression: a case report.}

Fecha de recepción: 17-01-2019

Fecha de aceptación: 17-04-2019
Carmela Martínez Vispo

Universidad de Santiago de Compostela

Ana López Durán

Universidad de Santiago de Compostela

Elisardo Becoña

Universidad de Santiago de Compostela

\section{resumen/alostract:}

Fumar es uno de los factores de riesgo más importantes para la calidad de vida, así como para la salud física y psicológica de las personas. La evidencia de la que disponemos muestra que el consumo de tabaco está estrechamente relacionado con la depresión. En el presente artículo presentamos la evaluación e intervención psicológica para dejar de fumar de una mujer de 49 años de edad con depresión y con un consumo diario de 15 cigarrillos. Se aplicó una intervención psicológica de tipo cognitivo-conductual para dejar de fumar con componentes de activación conductual para el manejo del estado de ánimo. Dicha intervención se llevó a cabo en ocho sesiones individuales. Tras la intervención, la persona dejó de fumar y se mantuvo abstinente en los seguimientos realizados a lo largo del año siguiente, mostrando también una notable mejora en la sintomatología depresiva tanto a corto como a largo plazo. A modo de conclusión, este tipo de intervención psicológica podría considerarse una opción adecuada para dejar de fumar y para el manejo del estado de ánimo, logrando una mejora en el bienestar y salud de las personas.

Smoking is the leading risk factor for quality of life, as well as for physical and psychological health. In fact, research has shown that tobacco use is closely related to depression. We present the assessment and intervention to quit smoking of a 49-year-old woman with depression and with a daily consumption of 15 cigarettes. A cognitive-behavioral intervention to quit smoking was applied with behavioral activation components for mood management. This intervention was carried out in eight individual sessions. At the end of the intervention, the participant quit smoking and remained abstinent at the follow-ups conducted throughout one year. She also showed a notable improvement in depressive symptomatology at short and long term after quitting. In conclusion, this intervention approach could be considered an adequate option to aid in the smoking cessation process and also to improve depressive symptoms, which implies a better quality of life and health.

\section{palabras clave/keywords:}

intervención psicológica, dejar de fumar, depresión.

psychological intervention, smoking cessation, depression.

\section{Introducción}

El consumo de tabaco es uno de los factores de riesgo más importantes para la salud, siendo en la actualidad un problema socio-sanitario de primer orden (U. S. Department of Health \& Human Services, 2014). Aunque en la mayoría de países desarrollados la prevalencia del 
consumo de tabaco sigue una tendencia descendente, todavía fuma una parte importante de la población. De hecho, los últimos datos de la Encuesta Nacional de Salud (Ministerio de Sanidad, Consumo y Bienestar Social, 2017), señala que el 22,1\% de la población de 15 y más años afirma que fuma a diario.

Además de las consecuencias que tiene el tabaco para la salud física y calidad de vida, también se ha relacionado con diferentes problemas psicopatológicos (Lawrence, Mitrou y Zubrick, 2009). Existen numerosos estudios que han encontrado una relación entre el consumo de tabaco y los trastornos del estado de ánimo, como la depresión (Mathew, Hogarth, Leventhal, Cook y Hitsman, 2017). Así, estudios epidemiológicos indican que las personas con depresión tienen el doble de probabilidades de fumar en comparación con aquellas sin depresión (Lê Cook et al., 2014). También existe evidencia que señala que las personas que fuman y que concurrentemente presentan sintomatología depresiva, tienen una mayor dependencia de la nicotina y un síndrome de abstinencia del tabaco más intenso (Grant, Hasin, Chou, Stinson y Dawson, 2004). Además, la sintomatología depresiva se ha relacionado con mayores dificultades para dejar de fumar, así como con un mayor riesgo de recaída temprana en el consumo (Cooper, Borland, McKee, Yong y Dugué, 2016).

En la actualidad existen intervenciones y tratamientos psicológicos eficaces para dejar de fumar (Siu, 2015). De hecho, la evidencia empírica muestra que el abordaje psicológico de la dependencia del tabaco mejora de manera substancial el logro de la abstinencia y su mantenimiento a largo plazo (Arias, García-Vera y Sanz, 2014; Patnode et al., 2015). También se ha encontrado que las intervenciones para dejar de fumar con componentes para el manejo del estado de ánimo son eficaces en personas con historia previa y/o actual de depresión (Gierisch, Bastian, Calhoun, McDuffie y Williams, 2012). Sin embargo, todavía es necesario seguir investigando qué componentes podrían ser más adecuados para este tipo de población.

Una técnica conductual que se ha propuesto para ser integrada en las intervenciones para dejar de fumar, en personas con sintomatología depresiva, es la Activación Conductual. Esta estrategia tiene como objetivo promover de manera estructurada conductas que permitan al individuo ponerse en contacto con reforzadores ambientales positivos contingentes a la conducta, a la vez que trata de bloquear las conductas de evitación (Dimidjian et al. 2011; Hopko et al. 2003). Para conseguir dicho objetivo las técnicas más usadas son el autorregistro y la programación de actividades, entre otras (Kanter et al. 2010). Tal estrategia ha demostrado ser una modalidad de tratamiento eficaz y efectiva para la depresión en diferentes muestras y contextos (Ekers, Webster, van Straten, Cuijpers, Richards y Gilbody, 2014). Pero, además, también ha resultado ser un procedimiento que obtiene buenos resultados cuando se ha integrado en el tratamiento de la dependencia del tabaco, así como en el consumo de otras sustancias (Martínez-Vispo, Martínez, López-Durán, Fernández del Río y Becoña, 2018).

El objetivo del presente estudio es la descripción del caso de una persona con depresión que acude a un tratamiento psicológico para dejar de fumar que tiene componentes específicos de activación conductual. 


\section{Método}

\section{Identificación del paciente}

E. es una mujer de 49 años de edad, que solicita intervención para dejar de fumar en la Unidad de Tabaquismo y Trastornos Adictivos de la Universidad de Santiago de Compostela. E. tiene estudios básicos, en la actualidad trabaja como limpiadora e informa tener un nivel socio-económico medio. Convive con su pareja, con la que está casada, y su hija, los cuales no fuman. En cuanto al consumo de tabaco, fuma una media de 15 cigarrillos al día con un contenido de $0,9 \mathrm{mg}$. de nicotina.

\section{Motivo de consulta}

E. acudió a la Unidad de Tabaquismo y Trastornos Adictivos después de que su endocrino le recomendase dejar de fumar. Anteriormente había intentado dejar de fumar en varias ocasiones, pero solo consiguió mantenerse abstinente un día entero durante el último año. Considera que fumar cigarrillos afecta en gran medida a su salud y refiere sentir molestias físicas relacionadas con el consumo de tabaco como fatiga, secreciones mucosas, mala circulación, migrañas frecuentes, dificultades para dormir y problemas respiratorios. Además, señala que experimenta un nivel muy elevado de ansiedad y malestar emocional en las ocasiones previas en las que ha intentado dejar de fumar por sí misma.

\section{Evaluación e Instrumentos}

En las sesiones de evaluación se llevó a cabo una entrevista clínica con el objetivo de evaluar variables relacionadas con el consumo de tabaco, intentos de abandono previos, procedimientos utilizados para dejar de fumar, apoyo social, consumo de alcohol, café, otras drogas y medicamentos, deseo de dejar de fumar y expectativas sobre el tratamiento

También se aplicó la entrevista de cribado de Episodio Depresivo Mayor (MDE; Muñoz, 1998). Se trata de una entrevista clínica estructurada formada por 18 ítems basados en los criterios diagnósticos del DSM-IV. Aunque no existe una versión basada en los criterios del DSM-5, la utilización de este instrumento puede servir para evaluar la ocurrencia de un episodio depresivo mayor actual o a lo largo de la vida.

Además se aplicaron los siguientes cuestionarios, los cuales han sido validados en población española y cuentan con características psicométricas adecuadas:

- Cuestionario sobre el Hábito de Fumar (Becoña, 1994). Este instrumento de 56 ítems recoge información sociodemográfica (p.ej., sexo, edad, nivel educativo) y sobre el consumo de tabaco (p.ej., numero de cigarrillos, marca de cigarrillos, años fumando).

- Escala para la Evaluación de los Estadios de Cambio (McConnaughy, Prochaska y Velicer, 1983). Se basa en el modelo transteórico de cambio (Prochaska, DiClemente y Norcross, 1992) y nos permite identificar en qué estadio de cambio se halla el fumador (precontemplación, contemplación, preparación para la acción).

- Escala de Tentación a Fumar (forma corta; Velicer, DiClemente, Rossi y Prochaska 1990). Consta de 9 ítems que evalúan tres dimensiones relacionadas con la tentación a fumar en ciertas situaciones: (1) en situaciones de afecto positivo/situación social, 
(2) en situaciones de afecto negativo, y (3) en situaciones relacionadas con el hábito/ craving.

- Cuestionario de Ansiedad Estado-Rasgo (STAI A-E y STAI A-R; Spielberger, Gorsuch y Lushene, 1982). Este instrumento está compuesta por 20 ítems y tiene dos componentes. El primero de ellos evalúa la tendencia del individuo a responder con ansiedad ante situaciones percibidas como amenazantes (ansiedad rasgo), mientras que el segundo hace referencia a un período transitorio donde está presente la sintomatología ansiosa, la cual puede variar en intensidad (ansiedad estado).

- Inventario de Depresión de Beck (BDI-II; Sanz y Vázquez, 2011). Está formado por 21 ítems. La puntación total puede oscilar entre 0 y 63 , siendo puntuaciones más altas las que indican mayor sintomatología depresiva.

Para comprobar de modo objetivo la abstinencia se usó el nivel de monóxido de carbono en aire espirado (CO), que se midió con el Micro+ Smokerslyzer ${ }^{\circledR}$. En la evaluación inicial, en cada una de las sesiones de intervención y en los seguimientos a los 3, 6 y 12 meses tras finalizar la intervención. La abstinencia autoinformada fue validada bioquímicamente con esta medida, considerándose confirmada con valores inferiores a 10 partículas por millón (p.p.m.) (West, Hajek, Stead y Stapleton, 2005).

También se llevaron a cabo autorregistros de la conducta de fumar (Becoña, 2007). Esta herramienta permite recoger información diaria acerca del cigarrillo fumado en cada momento. La persona debe recoger la hora en la que fuman cada cigarrillo, el placer experimentado (en una escala de 0 a 10 puntos) y la situación en la que se encuentra (p.ej., después de comer).

Además, se aplicó el Cuestionario de Evaluación Post-tratamiento (Becoña y Míguez, 1995), en la última sesión (sesión 8) Este instrumento evalúa aspectos referidos al consumo de cigarrillos o en el caso de que el participante dejase de fumar, la fecha de abandono y confianza en mantenerse abstinente en los próximos 6 meses (escala de 0 a 10). Otros aspectos evaluados son la percepción de mejoría/empeoramiento físico y psicológico desde el inicio del tratamiento y la satisfacción con los servicios recibidos.

Por último, se aplicaron Cuestionarios de Evaluación en los Seguimientos (Becoña y Míguez, 1995), los cuales se llevan a cabo al mes, a los 3, 6 y 12 meses después de la última sesión. Este instrumento tiene dos versiones en función del estatus de fumador del participante (fumador vs. abstinente). A través de este se recogen datos acerca del apoyo social percibido, intentos de abandono tras finalizar el tratamiento, tentaciones o impulsos a fumar, y ventajas de haber logrado dejar de fumar (versión para abstinentes).

\section{Historia del problema}

E. comenzó a fumar regularmente a los 18 años de edad, aunque ya desde los 16 años había fumado de manera ocasional. Por tanto, lleva 31 años fumando y, a lo largo de estos años, solo logró estar abstinente un día. Con respecto a la forma de fumar, señala que inhala el humo, da bastantes caladas a cada cigarrillo y los fuma prácticamente enteros. 
En cuanto al estado de ánimo, E. refiere estar a tratamiento para la depresión desde hace 8 años. La sintomatología depresiva comenzó tras la muerte de su madre, y se mantuvo durante este período debido a otros eventos entre los que destacan la muerte de un hermano y problemas de pareja. También informa de experimentar ansiedad elevada desde hace dos años, con dos episodios de ansiedad por los que ha recibido atención médica. El tratamiento actual que está recibiendo para la depresión es de tipo farmacológico (duloxetina y lorazepam). E. comenta que fumar cigarrillos le "ayuda a aliviar la ansiedad" y a "relajarse y desconectar de los problemas y las preocupaciones".

\section{Evaluación inicial}

E. obtuvo un nivel de CO de 16 p.p.m. A través de los autorregistros se observó que las situaciones en las que fuma con más frecuencia y tienen una valoración más positiva fueron después de comer y tras realizar tareas (ir al banco o después de trabajar). En las escalas que evalúan el deseo de dejar de fumar y el de realizar el programa para dejar de fumar, señala una puntuación de 10 (en una escala de 0 a 10 puntos). Además, en la evaluación de los estadios de cambio, se sitúa en el de "Preparación para la Acción", ya que tiene la intención de dejarlo en los próximos 30 días y ha conseguido estar sin fumar al menos un día en los últimos doce meses.

Por otro lado, en la Escala de Tentación a Fumar obtiene puntuaciones elevadas en dos de las tres subescalas, mostrando una tentación a fumar muy alta en situaciones relacionadas con el afecto negativo y con el afecto positivo. Mientras que en la subescala craving/hábito, la puntuación es moderada.

En cuanto a los datos obtenidos en la entrevista de screening para la detección de episodios depresivos, MDE, E. puntúa positivamente tanto para episodio depresivo mayor en el pasado como en el momento actual. Cabe destacar que E. refiere que esta sintomatología interfiere de manera significativa en su vida y actividades. Estos datos están en consonancia con las puntuaciones obtenidas en el BDI-II, en el que obtiene una puntuación de 37, lo que indica la presencia de sintomatología depresiva severa (Beck et al., 1996).

En el STAI Rasgo obtiene una puntuación directa de 36 que la sitúa en el percentil 85 (por encima de la media de la población general). Esta puntuación refleja que, en general, es una persona que tiende a reaccionar de manera ansiosa. En el STAI Estado su puntuación directa fue de 31 , percentil 75 , lo que indica que en el momento de la evaluación presentaba sintomatología ansiosa.

\section{Procedimiento}

Antes de comenzar, se realizó la explicación del protocolo de evaluación, intervención y seguimientos, y se firmó el consentimiento informado. En total se realizaron dos sesiones de evaluación, ocho sesiones de intervención y sesiones de seguimiento a los 3, 6 y 12 meses. Tanto en la evaluación como en cada una de las sesiones de intervención y seguimiento se realizaron evaluaciones del nivel de monóxido de carbono en aire espirado (CO), con el objetivo de proporcionar feedback y de tener una validación bioquímica de la abstinencia. En la última sesión de intervención E. cumplimentó un cuestionario de final de tratamiento, y en cada uno de los seguimientos también cubrió un cuestionario de seguimiento. 


\section{Resultados}

\section{Intervención psicológica}

La participante asiste a un tratamiento para dejar de fumar basado en técnicas cognitivoconductuales con componentes de activación conductual (Tabla 1), sin ayuda farmacológica para dejar de fumar. Consta de ocho sesiones de una hora de duración con una periodicidad semanal, que se describen a continuación, con los resultados obtenidos:

\section{Primera sesión}

En esta sesión se presenta cómo se van a estructurar las sesiones y se proporciona el material por escrito de las tareas a realizar durante la primera semana de intervención. Además, se le facilita una gráfica del consumo de cigarrillos, así como los autorregistros que debe cumplimentar diariamente.

Una de las estrategias que se utilizará en todas las sesiones es el feedback fisiológico a través de la medición del CO. Esta estrategia permite evaluar la reducción del consumo de manera objetiva. En esta primera sesión, E. obtuvo un valor de CO de 20 p.p.m.

Para establecer la línea base de consumo, se usaron los autorregistros realizados en días previos. Además, estos también permitieron realizar el análisis funcional de la conducta de fumar. Con ellos se explicó cuáles eran los antecedentes y consecuentes de fumar y cómo reconocerlos.

Durante esta primera sesión se le proporciona material por escrito con información acerca de la dependencia de la nicotina, datos objetivos sobre los componentes del tabaco, cuáles son las consecuencias negativas que produce en la salud y los beneficios de dejar de fumar. Asimismo, se discutieron los motivos por los que quiere dejar de fumar en ese momento, y cuáles son las razones por las que cree que seguía fumando.

A continuación, se le explicó la técnica de desvanecimiento. Esta consiste en la reducción gradual de la ingesta de nicotina y alquitrán de manera semanal, a través de cambios de marca de tabaco y reducción paulatina del número de cigarrillos. De esta forma, se consigue una reducción de la dependencia fisiológica. En el caso de E. se indicó que a partir del día siguiente debía fumar una marca de cigarrillos que contuviera $0,7 \mathrm{mg}$. de nicotina, y se estableció un máximo de 10 cigarrillos diarios. El hecho de realizar los autorregistros había reducido su consumo.

También se le sugirió que comunicase al menos a una persona cercana que iba a dejar de fumar en los próximos 30 días. Esta estrategia está destinada a conseguir un compromiso social en el proceso de abandono del tabaco.

Otras tareas que debía cumplir a lo largo de la semana fueron las siguientes: (1) reducir la profundidad de las inhalaciones al fumar, (2) dejar el último tercio del cigarrillo sin fumar, (3) no tener el cigarrillo en la boca (ni siquiera apagado) mientras realizaba otras tareas y, (4) no aceptar ofrecimientos de cigarrillos (norma orientada a la prevención de la recaída a través de la práctica de rechazar ofrecimientos). 


\section{Segunda sesión}

En primer lugar, se revisaron los autorregistros y tareas realizadas durante la semana anterior. E. había respetado el límite de 10 cigarrillos al día como máximo, aunque cuando se evaluó el nivel de CO, obtuvo 26 p.p.m. Este valor era superior al de la semana anterior, por lo que se le preguntó acerca de la forma de fumar (el incremento de CO puede producirse al cambiar la forma de fumar; por ejemplo, fumar rápido, inhalaciones más profundas o mantener el humo más tiempo en los pulmones). E. reconoció que esa semana había realizado caladas más profundas y largas. Entre las dificultades surgidas a lo largo de la semana destacó el cambio de marca, ya que había notado mucho la reducción de nicotina y que los cigarrillos no la "saciaban". También señaló que en ciertas situaciones de estrés y ansiedad tenía muchas dificultades para controlar las ganas de fumar.

Una vez finalizada la revisión de la semana, se estableció un nuevo cambio de marca. En esta ocasión E. fumaría una marca que contuviese $0,5 \mathrm{mg}$ de nicotina y se estableció hasta un máximo de 8 cigarrillos al día. En esta sesión se le explicó la técnica del control de estímulos, la cual tiene como objetivo debilitar las asociaciones establecidas entre fumar y ciertas situaciones, actividades, pensamientos o estados emocionales. Así, debía elegir tres situaciones en las que dejaría de fumar a partir del día siguiente. E. escogió no fumar en el coche, mientras caminaba y cuando bebiese alcohol.

Por otro lado, se explicaron diferentes estrategias para hacer frente a los síntomas del síndrome de abstinencia, como beber abundantemente, reducir el consumo de estimulantes como el café, reducir la ingesta de alcohol, realizar actividad física, tomar chicles o caramelos sin azúcar, etc.

Para finalizar la sesión, se introduce la técnica de la respiración profunda. Esta pretende conseguir una disminución de los niveles de activación del organismo. Como tarea para realizar en casa, se propone que practique esta técnica varias veces al día a lo largo de toda la semana. De esta forma conseguiría dominarla y poder usarla para manejar las ganas de fumar o en otras situaciones estresantes. Además, en su caso eran un claro antecedente de la conducta de fumar.

\section{Tercera sesión}

En la revisión de cómo transcurrió la semana previa, E. señala que le resultó difícil, pero más llevadera que la anterior. De hecho, E. indica que cumplió con el límite máximo de cigarrillos todos los días. También señaló que no había fumado en las situaciones que había escogido y que había conseguido dar caladas menos profundas. Estos logros se vieron reflejados en la evaluación del nivel de CO, ya que obtuvo un nivel de tan solo 9 p.p.m.

El nuevo límite de cigarrillos establecido para la siguiente semana fue de 7 cigarrillos al día de una marca de $0,3 \mathrm{mg}$. de nicotina. En esta sesión también debía escoger otras tres situaciones en las que dejar de fumar a partir del siguiente día. En esta ocasión escogió no fumar mientras veía la TV, hablaba por teléfono y en momentos de espera (p.ej., esperar el autobús).

Durante la sesión se trató el tema de la alimentación, el ejercicio físico y el incremento de peso asociado a dejar de fumar. Para E. era especialmente importante mantener el peso 
debido a sus problemas endocrinos. Se le indicaron una serie de estrategias básicas de alimentación y varias estrategias conductuales para controlar la ingesta (p.ej., comer despacio o ir a comprar después de haber comido).

Por último, se introdujo la técnica de activación conductual. En primer lugar, se explica la racionalidad de dicha estrategia y la relación entre las conductas que llevamos a cabo y el estado de ánimo. Se resaltó que el propósito final de esta técnica es aumentar las actividades que mejoran el ánimo y disminuir las que lo empeoran, por lo que como tarea para casa se le pide que haga un autorregistro de las actividades que realiza en su día a día (trabajo, ocio, lúdicas, etc.), con el objetivo de conocer qué actividades son reforzantes y cuáles no.

\section{Cuarta sesión}

Como en las anteriores sesiones, se comenzó con la revisión de las tareas semanales. En esta ocasión E. indica que tuvo muchas dificultades para cumplir el tope de cigarrillos, ya que la marca con bajo contenido en nicotina no la satisfacía. Sin embargo, afirma estar motivada y satisfecha ya que ha empezado a notar mejoras en la respiración, menor fatiga y una reducción de la intensidad de las migrañas que suele padecer. En esta ocasión, se cambia de nuevo de marca de cigarrillos, a una que contenga $0,1 \mathrm{mg}$. de nicotina, con un máximo de 5 cigarrillos diarios. Por último, respetó la tarea de no fumar en las situaciones acordadas. En la evaluación de $\mathrm{CO}$ obtuvo un nivel de 8 p.p.m.

Se llevó a cabo la revisión del autoregistro de actividades diarias para establecer la línea base de actividades reforzantes. En general E. no manifiesta dificultades para realizarlo, y comentó que, con esta tarea se dio cuenta que en su día a día había pocas actividades positivas, ya que solo trabajaba, hacía las tareas de casa y en alguna ocasión veía algún programa en la TV que le gustaba. Como tarea para casa se le propone que elabore una lista de conductas y actividades que le gustaría llevar a cabo y escoja dos de ellas para realizar durante la semana. Se le indica que debe seguir registrando sus actividades diarias indicando el nivel de satisfacción asociado a las mismas.

Por otro lado, se planteó la cuestión de verse como una no fumadora y se explicó la distinción entre caída y recaída. De esta forma, se pretende que comprenda mejor el proceso de recaída, y así poder evitarla en caso de que se produjese algún "desliz", una vez alcanzada la abstinencia.

También se explicó cómo detectar las tentaciones e impulsos a fumar y se analizaron las estrategias empleadas para manejarlos. E. indicó que tanto la respiración como distraerse con otra actividad le ayudaban a superar las ganas de fumar.

\section{Quinta sesión}

En la revisión de la semana, E. señaló que había manejado bien las ganas de fumar y que, como cada vez los cigarrillos la satisfacían menos, las tentaciones de fumar eran menos intensas. De hecho, consiguió cumplir con todas las tareas establecidas, obteniendo un nivel de $\mathrm{CO}$ de 5 p.p.m. Señaló que estaba muy motivada ya que estaba experimentando muchas mejoras físicas. En esta sesión se le plantea que siga con la marca de $0,1 \mathrm{mg}$. de nicotina, intente reducir a 4 cigarrillos al día y acuda a la siguiente sesión 24 horas sin fumar. E. ma- 
nifestó su preocupación por si sería capaz de conseguirlo, pero a la vez se mostró dispuesta a intentarlo. Para reforzar esta decisión nos centramos en las creencias erróneas que existen acerca del proceso de dejar de fumar.

Con respecto a las actividades relacionadas con la activación conductual, durante la revisión de las mismas señaló que le ayudaron a estar de mejor humor. E. había escogido ir al cine con su marido e ir a la peluquería, actividades que no realizaba desde hacía años y que le resultaron muy satisfactorias.

En esta sesión se introduce el concepto de las conductas de evitación, y cómo estas influyen en el estado de ánimo. E. reconoce que muchas veces se siente sin ganas de hacer nada, que ha rechazado muchas veces salir a tomar algo con amigas o realizar actividades, lo cual hace que finalmente se sienta peor. Se le refuerza la realización de dichas actividades y como tarea para casa realizar otras dos actividades de su listado durante la semana.

Al final de la sesión se abordan los conceptos de estrés y ansiedad, y su relación con el tabaco. E. reconoce que ha fumado en muchas ocasiones para "relajarse cuando estaba nerviosa", aunque reconoce que no es eficaz. Por último, se le entrenó en la técnica de solución de problemas, con el objetivo de mejorar la toma de decisiones ante situaciones difíciles y así evitar que en el futuro utilice el tabaco como estrategia de afrontamiento.

\section{Sexta sesión}

E. acudió a la sesión sin haber fumado durante cinco días. Comentó que cuando se le acabó la cajetilla decidió no comprar ese día cigarrillos y, al ver que se encontraba bien y sin ansiedad, decidió seguir así. Reconoció que en ciertos momentos del día tenía ganas de fumar y que los impulsos eran fuertes en algunas ocasiones, pero mucho más manejables de lo que esperaba. En la evaluación de CO obtuvo 2 p.p.m., lo cual la animó a seguir abstinente. Manifestó también que estaba experimentando muchas mejoras físicas a todos los niveles y que haberlo logrado también le hacía sentirse contenta y con una mayor autoestima. Además, las personas de su entorno la estaban apoyando y animando a seguir sin fumar, lo que la reforzaba mucho.

Se revisó la realización de las actividades de la semana, E. señala que en esta ocasión quedó con unas amigas para salir a caminar y se había comprado una novela. Comentó que la realización de estas actividades la distraía y hacía que estuviese más animada. Se le propuso que esta semana siguiese llevando a cabo actividades de ese tipo y registrándolas.

En esta sesión se introdujo el concepto de rumiación y cómo los pensamientos influyen en las emociones y las conductas que llevamos a cabo. Nos centramos en la identificación de dichos pensamientos y en estrategias que pueden ser útiles para detenerlos.

\section{Séptima sesión}

E. acudió a la sesión sin haber fumado ni una sola calada desde hacía 12 días, obteniendo en la evaluación del CO 2 p.p.m. Señala que el proceso había sido más fácil de lo que ella esperaba, aunque en ciertos momentos las ganas de fumar aparecían con mucha intensidad. Éstas estaban relacionadas con situaciones estresantes o que le generaban ansiedad, por lo que se insiste en la práctica de la respiración profunda y de otras estrategias de sesiones an- 
teriores. A pesar de esto, se muestra muy satisfecha por haberlo logrado. Con respecto a las actividades, afirma que estas le han ayudado a mejorar su estado de ánimo.

Durante la sesión se refuerza su implicación en el proceso de dejar de fumar, así como por la realización de las actividades propuestas. Finalmente se realiza un breve resumen y recordatorio de conceptos (especialmente la distinción entre caída y recaída) y estrategias (técnica de respiración, identificación de situaciones de estrés, programación de actividades, resolución de problemas, etc.) explicados durante las semanas anteriores.

\section{Octava sesión}

Acude a esta última sesión sin haber fumado desde hacía 19 días, por lo que manifiesta sentirse muy orgullosa y satisfecha. En la medición de CO obtiene 2 p.p.m. Durante esta sesión se habló de las consecuencias positivas relacionadas con dejar de fumar y se realizó un balance acerca de las ventajas y desventajas de mantenerse sin fumar a largo plazo. Asimismo, se repasaron los conceptos de caída y recaída, y se analizaron de nuevo las tentaciones o impulsos de fumar y aquellas estrategias que estaba aplicando para superarlas. También se abordó la importancia de mantenerse activa y realizar aquellas actividades que lograban una mejoría en el ánimo. Se reforzó la idea de que emplease lo aprendido en otros ámbitos de su vida cotidiana.

Finalmente, cumplimentó el Cuestionario de Evaluación Final de Tratamiento (Becoña, 1994), y el cuestionario BDI-II. E. señaló sentirse muy satisfecha por haber conseguido dejar de fumar y manifestó una total confianza en mantenerse sin fumar. Entre los beneficios destaca haber experimentado más energía, una mejoría en la respiración y en la percepción de sabores y olores. Además, señaló que a nivel psicológico se encontraba mejor, ya que había logrado algo que pensaba que era imposible, por lo que su autoestima también había mejorado. La puntuación obtenida en el BDI-II se redujo, pasando de 37 en la evaluación inicial (sintomatología severa) a 14 (sintomatología leve) en esta última sesión de intervención (ver Tabla 2).

\section{Seguimiento}

Durante los seguimientos presenciales realizados a los 3, 6 y 12 meses, E. indicó que se mantuvo sin fumar ni una sola calada desde la última sesión de intervención. Dicha abstinencia se validó bioquímicamente con la evaluación del $\mathrm{CO}$, tal y como puede observarse en la Figura 1. Señala que, en ciertos momentos de estrés, o cuando está con personas que fuman, es cuando aparecen las tentaciones o impulsos de fumar. Sin embargo, comenta que puede manejarlas sin mucha dificultad. En cuanto a las mejoras experimentadas, señala que se encuentra mucho mejor a nivel físico y psicológico (ej., menos cansancio, mejor capacidad respiratoria y una mejora en el estado de ánimo) (ver Figura 2).

\section{Discusión y conclusiones}

El objetivo de este estudio de caso era realizar una descripción de una intervención cognitivo-conductual para dejar de fumar con componentes de activación conductual en una persona con sintomatología depresiva elevada. Para ello se ha descrito el motivo de la intervención, la historia del problema, el proceso de evaluación, así como los resultados obtenidos 
Tabla 1.- Componentes del tratamiento cognitivo-conductual para dejar de fumar y activación conductual

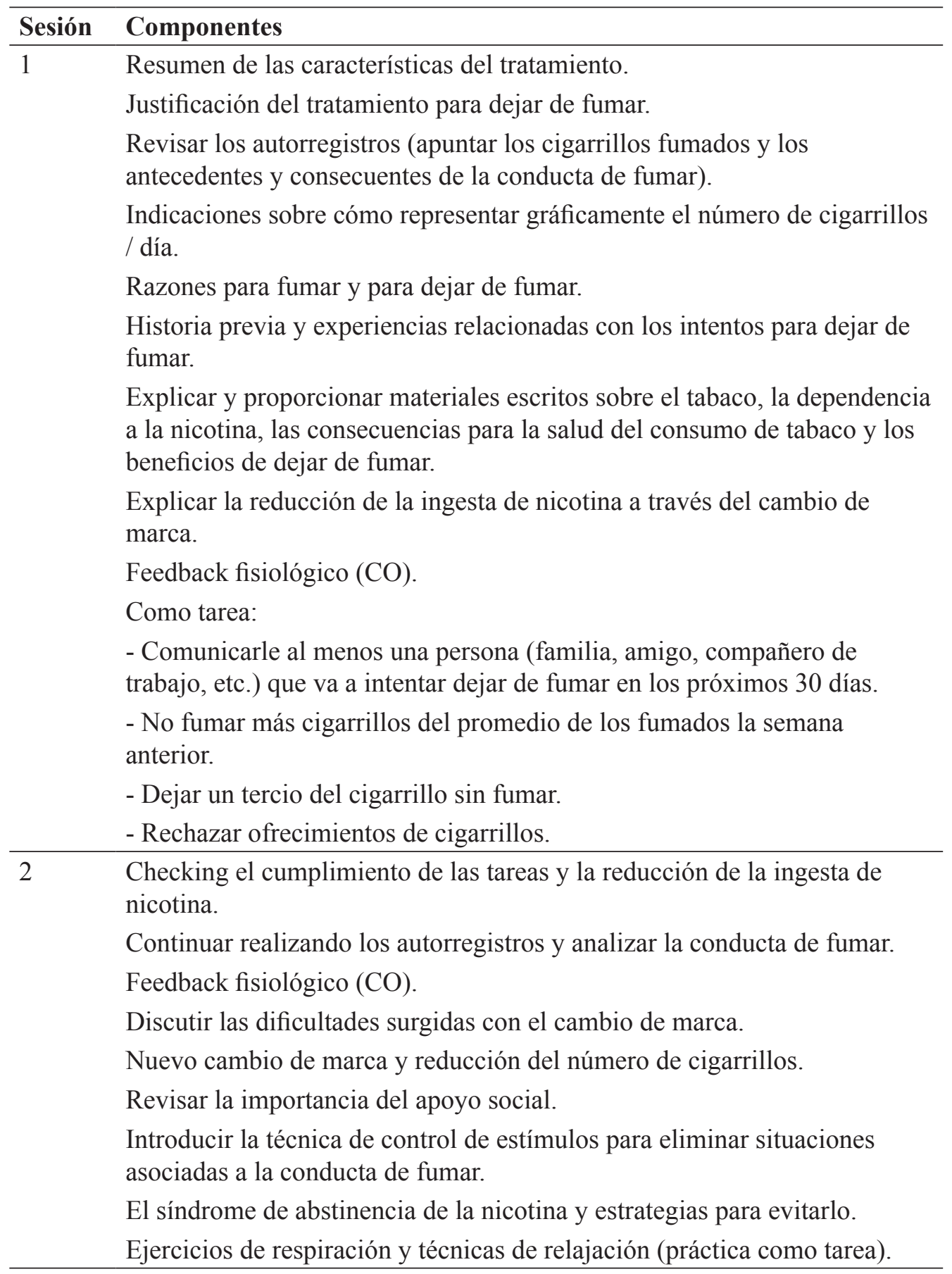


3 Checking la reducción de la ingesta de nicotina, la reducción del consumo de cigarrillos y el cumplimiento del control de estímulos.

Checking del cumplimiento de los ejercicios de respiración y estrategias para evitar los síntomas del síndrome de abstinencia.

Feedback fisiológico (CO)

Continuar realizando los autorregistros y analizar la conducta de fumar.

Nuevo cambio de marca y reducción del número de cigarrillos.

Continuar con la técnica de control de estímulos.

Explicar y proporcionar materiales escritos para el control de peso y para promover el ejercicio físico.

Continuar con los ejercicios de respiración y las estrategias para evitar los síntomas de abstinencia.

Justificación de la influencia del estado de ánimo en el abandono del hábito de fumar (materiales escritos).

Para casa: autorregistro de actividades diarias.

$4 \quad$ Checking de la reducción de la ingesta de nicotina, la reducción del consumo de cigarrillos y el cumplimiento del control de estímulos.

Checking del cumplimiento de los ejercicios de respiración y estrategias para evitar los síntomas del síndrome de abstinencia.

Feedback fisiológico (CO).

Continuar realizando los autorregistros y analizar la conducta de fumar.

Nuevo cambio de marca y reducción del número de cigarrillos.

Continuar con la técnica de control de estímulos.

Estrategias de manejo del estrés y la ansiedad.

Revisión del autorregistro de actividades y reconocimiento de patrones conductuales relacionados con el estado de ánimo, y la forma en que participar en actividades gratificantes e importantes puede afectar su estado de ánimo general.

Tarea: continuar con la programación de actividades, crear una lista de actividades placenteras y elegir dos para hacer durante la semana 
$5 \quad$ Ckecking de la reducción de la ingesta de nicotina, la reducción del consumo de cigarrillos y el cumplimiento del control de estímulos.

Feedback fisiológico (CO).

Continuar realizando los autorregistros y analizar la conducta de fumar.

Nuevo cambio de marca y reducción del número de cigarrillos.

Manejo de la ansiedad y la ira.

Autorrefuerzo.

Discusión acerca de conceptos erróneos relacionados con el tabaco.

Entrenamiento para resolver problemas.

Reconocer el comportamiento de evitación y el impacto en el estado de ánimo.

Programación de actividades y participación en 2 actividades agradables / semana.

6 La experiencia de dejar de fumar y los síntomas del síndrome de abstinencia.

Feedback fisiológico (CO).

Discutir y planificar estrategias para situaciones de alto riesgo de caída o recaída. Factores motivadores para mantener la abstinencia.

Beneficios de dejar de fumar.

Barreras comunes para mantener la abstinencia.

Pensamientos de rumiación, el proceso para dejar de fumar y la recaída.

Checking de las tareas programadas.

Programación de actividades para la próxima semana y participación en dos actividades agradables por semana.

$7 \quad$ La experiencia de dejar de fumar y los síntomas del síndrome de abstinencia.

Feedback fisiológico (CO).

Discutir y planificar estrategias para situaciones de alto riesgo de caída o recaída.

Factores motivadores para mantener la abstinencia.

Beneficios de dejar de fumar.

Revisión de las estrategias de activación conductual y su influencia en el estado de ánimo.

Revisión de las conductas de evitación y los pensamientos de rumiación. Estrategias de prevención de la recaída. 
Feedback fisiológico (CO).

Gestionando el futuro como ex fumadores.

Fomento del mantenimiento de la abstinencia.

Estrategias a usar en caso de caídas o recaídas.

Revisar los factores motivadores, los cambios en el estilo de vida, la mejora de la salud física y psicológica al dejar de fumar.

Revisar estrategias de activación conductual.

Conclusión y manejo de posibles obstáculos para mantenerse sin fumar.

Tabla 2.- Cambio en las puntuaciones obtenidas en la evaluación de los síntomas depresivos (BDI-II) y de la tentación a fumar

Pretratamiento Postratamiento

Síntomas depresivos (BDI-II)

Escala de Tentación a fumar

Situaciones de afecto positivo/social

Situaciones de afecto negativo

Situaciones de hábito/craving
37

14

9

0

12

3

8

en las escalas de autoinforme aplicadas, las ocho sesiones de intervención y los datos de los seguimientos realizados a lo largo de un año tras finalizar la intervención.

Tal y como se ha señalado previamente, la sintomatología depresiva es una variable clave que influye en el proceso de dejar de fumar y que puede constituir una barrera para lograr la abstinencia (Cooper et al., 2016). De hecho, diversos estudios han señalado que la sintomatología depresiva está relacionada con una mayor probabilidad de recaída, así como una sintomatología más severa del síndrome de abstinencia y del craving (Reid y Ledgerwood 2016; Stepankova et al. 2017). Por ello, el desarrollo y la implementación de tratamientos específicos que aborden dicha sintomatología durante el proceso de dejar de fumar son claves para mejorar las tasas de abstinencia. La activación conductual es una intervención prometedora, ya que estudios previos indican que no solo es eficaz, sino también coste-efectiva en el tratamiento de la depresión (Eckers et al., 2014; Richards et al., 2016). Además, se trata de una intervención que favorece la realización de actividades reforzantes alternativas al consumo de cigarrillos, lo que hace que aborde aspectos relacionados no solo con el 
Figura 1.- Evolución del consumo de tabaco ( $\mathrm{n}^{\mathrm{o}}$ de cigarrillos/día) y nivel de monóxido de carbono $(\mathrm{CO})$ en aire espirado en partículas por millón en la evaluación, sesiones de intervención y seguimientos.

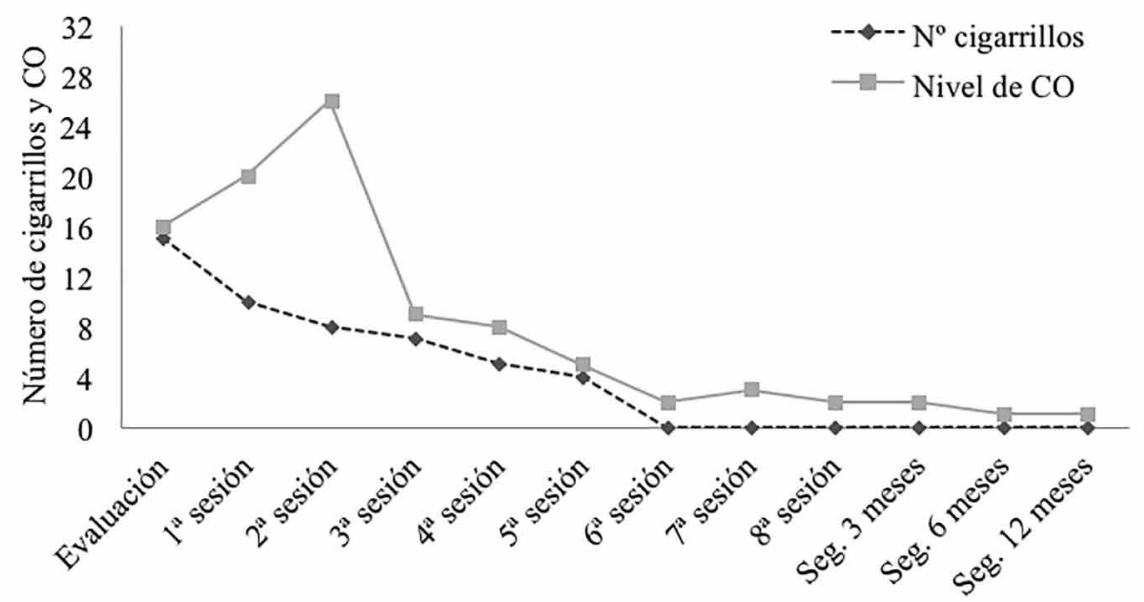

Sesiones y seguimientos

Figura 2.- Evolución de la sintomatología depresiva evaluada con el BDI-II: evaluación inicial, 1 final del tratamiento y seguimientos a los 3, 6 y 12 meses.

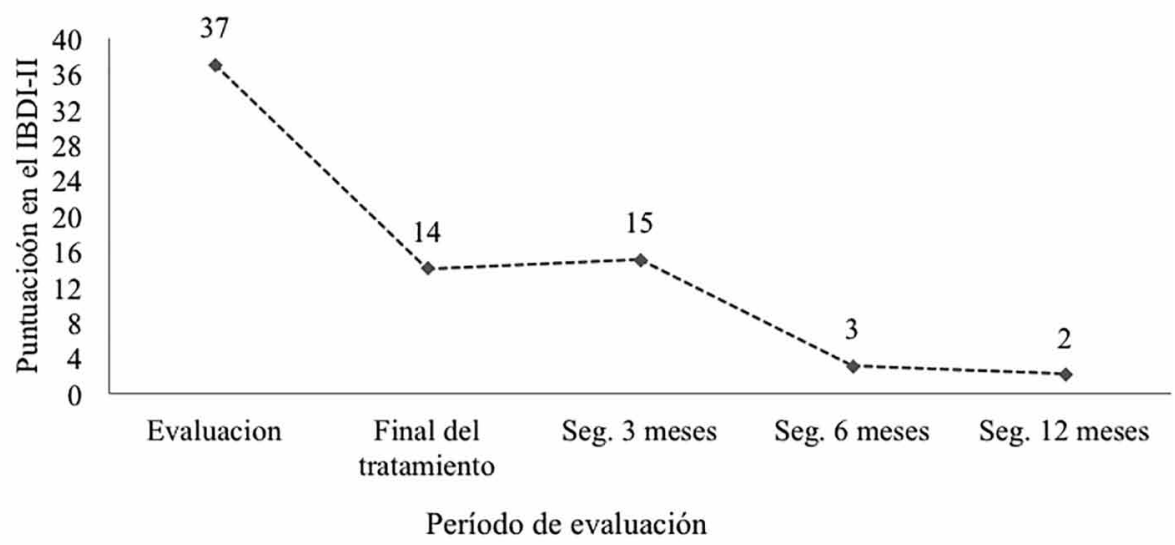

estado de ánimo sino también con los procesos de reforzamiento implicados en el inicio y mantenimiento de la conducta de fumar (Higgins, Heil y Lussier, 2004).

A pesar de la relevancia de los datos presentados, al tratarse de un estudio de caso, la capacidad de generalizar dichos resultados a toda la población de fumadores con sintomatología 
depresiva es limitada. Sin embargo, podemos concluir que una intervención cognitivo conductual para dejar de fumar, con componentes de activación conductual para el manejo del estado de ánimo, resulta útil para lograr y mantener la abstinencia a largo plazo y conseguir una mejora en el estado de ánimo. Estos datos revelan la necesidad de seguir investigando la eficacia de las intervenciones cognitivo-conductuales, especialmente en aquellas poblaciones más vulnerables o con características que pueden influir en el proceso de abandono del tabaco.

Este estudio de caso, como otros ensayos clínicos, revisiones y meta-análisis muestran que el tratamiento psicológico es un tratamiento de primera elección para el abordaje de la dependencia del tabaco (Siu et al., 2015). Hacerlo más accesible a los fumadores facilitaría la reducción de los niveles de morbi-mortalidad que produce fumar cigarrillos. En el caso de su combinación con la activación conductual, también logra mejorar el estado de ánimo y la reducción de la sintomatología depresiva, la cual está asociada no solo a fumar, sino también a mayores dificultades para lograr y mantenerse abstinente.

\section{Referencias}

Arias, I. G. F., García-Vera, M. P. y Sanz, J. (2014). Cuanta más psicología, mejor: eficacia para dejar de fumar de la terapia cognitiva conductual intensiva y de los parches de nicotina combinados con terapia cognitiva conductual intensiva y menos intensiva: Primer premio de la XX edición del Premio de Psicología Aplicada "Rafael Burgaleta" 2013. Clínica y Salud, 25, 1-10. doi: 10.5093/cl2014a7

Beck, A., Steer, R. A. y Brown, G. K. (1996). BDI-II. Beck Depression Inventory-Second Edition. Manual. San Antonio, TX: The Psychological Corporation.

Becoña, E. (1994). Evaluación de la conducta de fumar. En J. L. Graña (Ed.), Conductas Adictivas: Teoría, evaluación y tratamiento (pp. 403-454). Madrid: Debate.

Becoña, E. (2007). Programa para Dejar de Fumar. Vigo: Nova Galicia Edicións.

Becoña, E. y Míguez, M. C. (1995). Abstinencia, recaída y no abandono en un programa para dejar de fumar, Adicciones, 7, 41-58.

Cooper, J., Borland, R., McKee, S. A., Yong, H. H. y Dugué, P. A. (2016). Depression motivates quit attempts but predicts relapse: differential findings for gender from the International Tobacco Control Study. Addiction, 111(8), $1438-1447$.

Dimidjian, S., Barrera, M., Martell, C., Muñoz, R. F. y Lewinsohn, P. M. (2011). The origins and current status of behavioral activation treatments for depression. Annual Review of Clinical Psychology, 7(1), 1-38. doi:10.1146/ annurev-clinpsy-032210-104535

Ekers, D., Webster, L., Van Straten, A., Cuijpers, P., Richards, D. y Gilbody, S. (2014). Behavioural activation for depression; an update of meta-analysis of effectiveness and sub group analysis. PloS one, 9(6), e100100.

Gierisch, J. M., Bastian, L. A., Calhoun, P. S., McDuffie, J. R. y Williams, J. W. (2012). Smoking cessation interventions for patients with depression: a systematic review and meta-analysis. Journal of General Internal Medicine, 27,351-360. doi:10.1007/s11606-011-1915-2

Grant, B. F., Hasin, D. S., Chou, S. P., Stinson, F. S. y Dawson, D. A. (2004). Nicotine dependence and psychiatric disorders in the United States: results from the national epidemiologic survey on alcohol and related conditions. Archives of General Psychiatry, 61, 1107-1115. doi:61/11/1107

Higgins, S. T., Heil, S. H. y Lussier, J. P. (2004). Clinical implications of reinforcement as a determinant of substance use disorders. Annual Review of Psychology, 55, 431-461. 
Hopko, D. R., Lejuez, C. W., Ruggiero, K. J. y Eifert, G. H. (2003). Contemporary behavioral activation treatments for depression: Procedures, principles, and progress. Clinical Psychology Review, 23(5), 699-717. doi:10.1016/ S0272-7358(03)00070-9

Kanter, J. W., Manos, R. C., Bowe, W. M., Baruch, D. E., Busch, A. M. y Rusch, L. C. (2010). What is behavioral activation? A review of the empirical literature. Clinical Psychology Review, 30(6), 608-620. doi:10.1016/j.cpr.2010.04.001

Lawrence, D., Mitrou, F. y Zubrick, S. R. (2009). Smoking and mental illness: results from population surveys in Australia and the United States. BMC Public Health, 9(1), 285. doi: 10.1186/1471-2458-9-285

Lê Cook, B., Wayne, G. F., Kafali, E. N., Liu, Z., Shu, C. y Flores, M. (2014). Trends in smoking among adults with mental illness and association between mental health treatment and smoking cessation. JAMA, 311, 172-182. doi:10.1001/jama.2013.284985

Martínez-Vispo, C., Martínez, Ú., López-Durán, A., Fernández del Río, E. F. y Becoña, E. (2018). Effects of behavioural activation on substance use and depression: a systematic review. Substance Abuse Treatment, Prevention, and Policy, 13(1), 36. doi: 10.1186/s13011-018-0173-2

Mathew, A. R., Hogarth, L., Leventhal, A. M., Cook, J. W. y Hitsman, B. (2017). Cigarette smoking and depression comorbidity: systematic review and proposed theoretical model. Addiction, 112(3), 401-412.doi: 10.1111/add.13604

McConnaughy, E. A., Prochaska, J. O. y Velicer, W. F. (1983). Stages of change in psychotherapy: Measurement and sample profiles. Psychotherapy: Theory, Research, and Practice, 20, 368-375. doi: 10.1037/h0090198

Ministerio de Sanidad, Consumo y Bienestar Social (2017). Encuesta Nacional de Salud - Año 2017. Madrid: Ministerio de Sanidad, Consumo y Bienestar Social. Recuperado de https://www.mscbs.gob.es/estadEstudios/ estadisticas/encuestaNacional/encuestaNac2017/ENSE2017_notatecnica.pdf

Patnode, C. D., Henderson, J. T., Thompson, J. M., Senger, C. A., Fortmann, S. P. y Whitlock, E. P. (2015). Behavioral counseling and pharmacological interventions for tobacco cessation in adults, including pregnant women: A review of reviews for the U. S. Preventive Services Task Force. Annals of Internal Medicine, 163, 608-621. doi: 10.7326/M15-0171

Prochaska, J. 0., DiClemente, C. C. y Norcross, J. C. (1992). In search of the structure of change. In Self change (pp. 87-114). Springer, New York, NY.

Richards, D. A., Ekers, D., McMillan, D., Taylor, R. S., Byford, S., Warren, F. C., ... O'Mahen, H. (2016). Cost and outcome of behavioural activation versus cognitive behavioural therapy for depression (COBRA): a randomised, controlled, non-inferiority trial. The Lancet, 388(10047), 871-880.

Sanz, J. y Vázquez, C., (2011). BDI-II: Inventario de Depresión de Beck-II: Manual. Pearson, Madrid.

Siu, A. L. (2015). Behavioral and pharmacotherapy interventions for tobacco smoking cessation in adults, including pregnant women: U.S. preventive services task force recommendation statement. Annals of Internal Medicine, 163, 622-634. doi:10.7326/M15-2023

Spielberger, C. D., Gorsuch, R. L. y Lushene, R. (1982). Manual del Cuestionario de Ansiedad Estado-Rasgo (STAI). Madrid: TEA Ediciones.

Stepankova, L., Kralikova, E., Zvolska, K., Pankova, A., Ovesna, P., Blaha, M. y Brose, L. S. (2017). Depression and smoking cessation: evidence from a smoking cessation clinic with 1-year follow-up. Annals of Behavioral Medicine, 51(3), 454-463. doi:10.1007/s12160-016-9869-6

U. S. Department of Health \& Human Services. (2014). The health consequences of smoking-50 years of progress. A report of the Surgeon General. Rockville, MD: U. S. Department of Health and Human Services, Public Health Services, Office of the Surgeon General.

Velicer, W. F., DiClemente, C. C., Rossi, J.S. y Prochaska, J. 0. (1990). Relapse situations and self-efficacy: An integrative model. Addictive Behaviors, 15, 271-283. doi: 10.1016/0306-4603(90)90070-E

Velicer, W. F., Prochaska, J. O., Rossi, J. S. y Snow, M. G. (1992). Assessing outcome in smoking cessation studies. Psychological Bulletin, 111, 23-41.

West, R., Hajek, P., Stead, L. y Stapleton, J. (2005). Outcome criteria in smoking cessation trials: Proposal for a common standard. Addiction, 100, 299-303. doi: 10.1111/j.1360-0443.2004.00995.x 